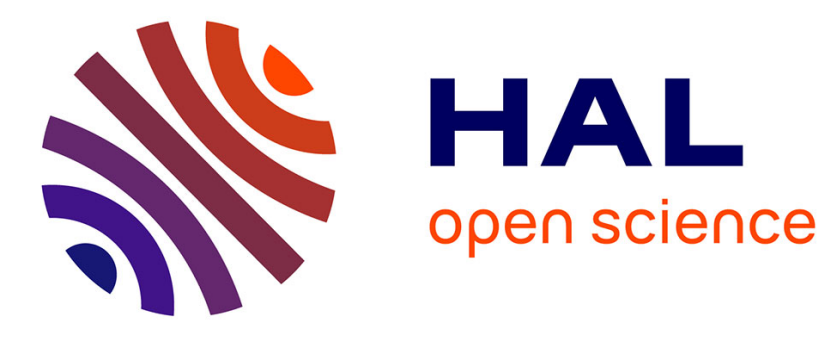

\title{
Sensor-Based Control Architecture for a Car-Like Vehicle
}

Christian Laugier, Thierry Fraichard, Philippe Garnier, Igor Paromtchik, Alexis Scheuer

\section{- To cite this version:}

Christian Laugier, Thierry Fraichard, Philippe Garnier, Igor Paromtchik, Alexis Scheuer. SensorBased Control Architecture for a Car-Like Vehicle. RR-3552, INRIA. 1998. inria-00073131

\section{HAL Id: inria-00073131 https://hal.inria.fr/inria-00073131}

Submitted on 24 May 2006

HAL is a multi-disciplinary open access archive for the deposit and dissemination of scientific research documents, whether they are published or not. The documents may come from teaching and research institutions in France or abroad, or from public or private research centers.
L'archive ouverte pluridisciplinaire HAL, est destinée au dépôt et à la diffusion de documents scientifiques de niveau recherche, publiés ou non, émanant des établissements d'enseignement et de recherche français ou étrangers, des laboratoires publics ou privés. 


\section{Sensor-Based Control Architecture for a Car-Like Vehicle}

Christian Laugier, Thierry Fraichard, Philippe Garnier,

Igor Paromtchik and Alexis Scheuer

$$
\mathrm{N}^{\circ} 3552
$$

Octobre 1998

THÈME 3 



\title{
RIN RIA
}

\section{Sensor-Based Control Architecture for a Car-Like Vehicle}

\author{
Christian Laugier, Thierry Fraichard, Philippe Garnier, \\ Igor Paromtchik and Alexis Scheuer \\ Thème 3 - Interaction homme-machine, \\ images, données, connaissances \\ Projet Sharp \\ Rapport de recherche $\mathrm{n}^{\circ} 3552$ - Octobre 1998 - 27 pages
}

\begin{abstract}
This report presents a control architecture endowing a car-like vehicle moving in a dynamic and partially known environment with autonomous motion capabilities. Like most recent control architectures for autonomous robot systems, it combines three functional components: a set of basic real-time skills, a reactive execution mechanism and a decision module. The main novelty of the architecture proposed lies in the introduction of a fourth component akin to a meta-level of skills: the sensor-based manouvres, i.e. general templates that encode high-level expert human knowledge and heuristics about how a specific motion task is to be performed. The concept of sensor-based manœuvres permit to reduce the planning effort required to address a given motion task, thus improving the overall response-time of the system, while retaining the good properties of a skill-based architecture, i.e. robustness, flexibility and reactivity. The report focuses on the trajectory planning function (which is an important part of the decision module) and two types of sensor-based manœuvres, trajectory following and parallel parking, that have been implemented and successfully tested on a real automatic car-like vehicle placed in different situations.
\end{abstract}

Key-words: motion autonomy, control architecture, car-like vehicle.

(Résumé : tsvp)

Unité de recherche INRIA Rhône-Alpes

655, avenue de l'Europe, 38330 MONTBONNOT ST MARTIN (France)

Téléphone : 0476615200 - International: +33476615200

Télécopie : 0476615252 - International: +33476615252 


\section{Une architecture de contrôle basé-capteurs pour un véhicule de type voiture}

Résumé : Ce rapport décrit une architecture de contrôle visant à doter un véhicule de type voiture de capacités de mouvement autonome. Comme un certain nombre d'architectures de contrôle récentes, elle combine trois composants fonctionnels: un ensemble "d'aptitudes" (où fonctions temps-réel de base), un mécanisme réactif d'exécution et un module de décision. La principale originalité de l'architecture proposée est l'introduction d'un méta-niveau d'aptitudes: les manœuvres basées-capteurs, i.e. des schémas généraux qui codent des connaissances de haut niveau expertes et heuristiques sur la façon de mener à bien un déplacement donné. Le concept de manœuvres basées-capteurs permet de réduire l'effort de planification exigé pour effectuer un déplacement donné et donc de diminuer les temps de réponse du système tout en conservant par ailleurs les bonnes propriétés d'une architecture reposant sur la notion d'aptitudes, i.e. la robustesse, la flexibilité et la réactivité. Le rapport se concentre sur la fonction de planification de trajectoire (qui est un composant important de l'architecture) et sur deux types de manœuvre basée-capteurs: le suivi de trajectoire et le créneau. Ces deux manœuvres ont été implantées et testées avec succès sur voiture réelle.

Mots-clé : autonomie de mouvement, architecture de contrôle, voiture. 


\section{Contents}

1 Introduction 5

2 Overview of the Control Architecture 5

2.1 Mission Monitor . . . . . . . . . . . . . . . . . . . . 7

2.2 Motion Controller . . . . . . . . . . . . . . . . . . 7

3 Model of the Vehicle $\quad 8$

4 Trajectory Planning 9

4.1 Outline of the Approach . . . . . . . . . . . . . . . . . 9

4.2 Path Planning . . . . . . . . . . . . . . . . . . . . . . 10

4.3 Velocity Planning . . . . . . . . . . . . . . . . . . . . . . 11

5 Sensor-Based Manœuvres 13

5.1 Trajectory Following . . . . . . . . . . . . . . . . . . . . 13

5.1 .1 Trajectory Tracking . . . . . . . . . . . . . . . . . . . . . 14

5.1 .2 Lane Changing . . . . . . . . . . . . . . . . . . . 15

5.2 Parallel Parking. . . . . . . . . . . . . . . . . . 16

6 Experimental Results $\quad 20$

$\begin{array}{lll}7 & \text { Related Works } & 23\end{array}$

8 Conclusion $\quad 24$

$\mathrm{RR} \mathrm{n}^{\circ} 3552$ 


\section{Introduction}

Autonomy in general and motion autonomy in particular has been a long standing issue in Robotics. In the late sixties-early seventies, Shakey [21], was one of the first robot able to move and perform simple tasks autonomously. Ever since, many authors have proposed control architectures to endow robot systems with various autonomous capabilities. Some of these architectures are reviewed in $\S 7$ and compared to the one presented in this report. These approaches differ in several ways, however it is clear that the control structure of an autonomous robot placed in a dynamic and partially known environment must have both deliberative and reactive capabilities. In other words, the robot should be able to decide which actions to carry out according to its goal and current situation; it should also be able to take into account events (expected or not) in a timely manner.

The control architecture presented in this report aims at meeting these two requirements. It is designed to endow a car-like vehicle moving on the road network with motion autonomy and was developed in the framework of the French Praxitèle programme aimed at the development of a new urban transportation system based on a fleet of electric vehicles with autonomous motion capabilities [22]. The road network is a complex environment, it is partially known and highly dynamic with moving obstacles (other vehicles, pedestrians, etc.) whose future behaviour is not known in advance. However the road network is a structured environment with motion rules (the highway code) and it is possible to take advantage of these features in order to design a control architecture that is efficient, robust and flexible.

The control architecture is presented in this report as follows: in the next section, the rationale of the architecture and its main features are overviewed: in particular, it introduces the key concept of sensor-based manouvres, i.e. general templates that encode the knowledge of how a specific motion task is to be performed. The model of the car-like vehicle that is used throughout the report is then described $(\S 3)$. One important component of the architecture is the trajectory planner whose purpose is to determine the trajectory leading the vehicle to its goal. Trajectory planning for car-like vehicles in dynamic environments remains an open problem and a practical solution to this intricate problem is presented in $\S 4$. Afterwards the concept of sensor-based manœuvres is explored in $\S 5$ and two types of manœuvres are presented in detail. These two manœuvres have been implemented and successfully tested on an experimental vehicle, the results of these experiments are finally presented in $\S 6$.

\section{Overview of the Control Architecture}

The control architecture is depicted in Fig. 1. It relies upon the concept of sensor-based manœuvres (SBM) which is derived from the Artificial Intelligence concept of script [27]. A script is a general template that encodes procedural knowledge of how a specific type of task is to be performed. A script is fitted to a specific task through the instantiation of variable parametres in the template; these parameters can come from a variety of sources (a priori 


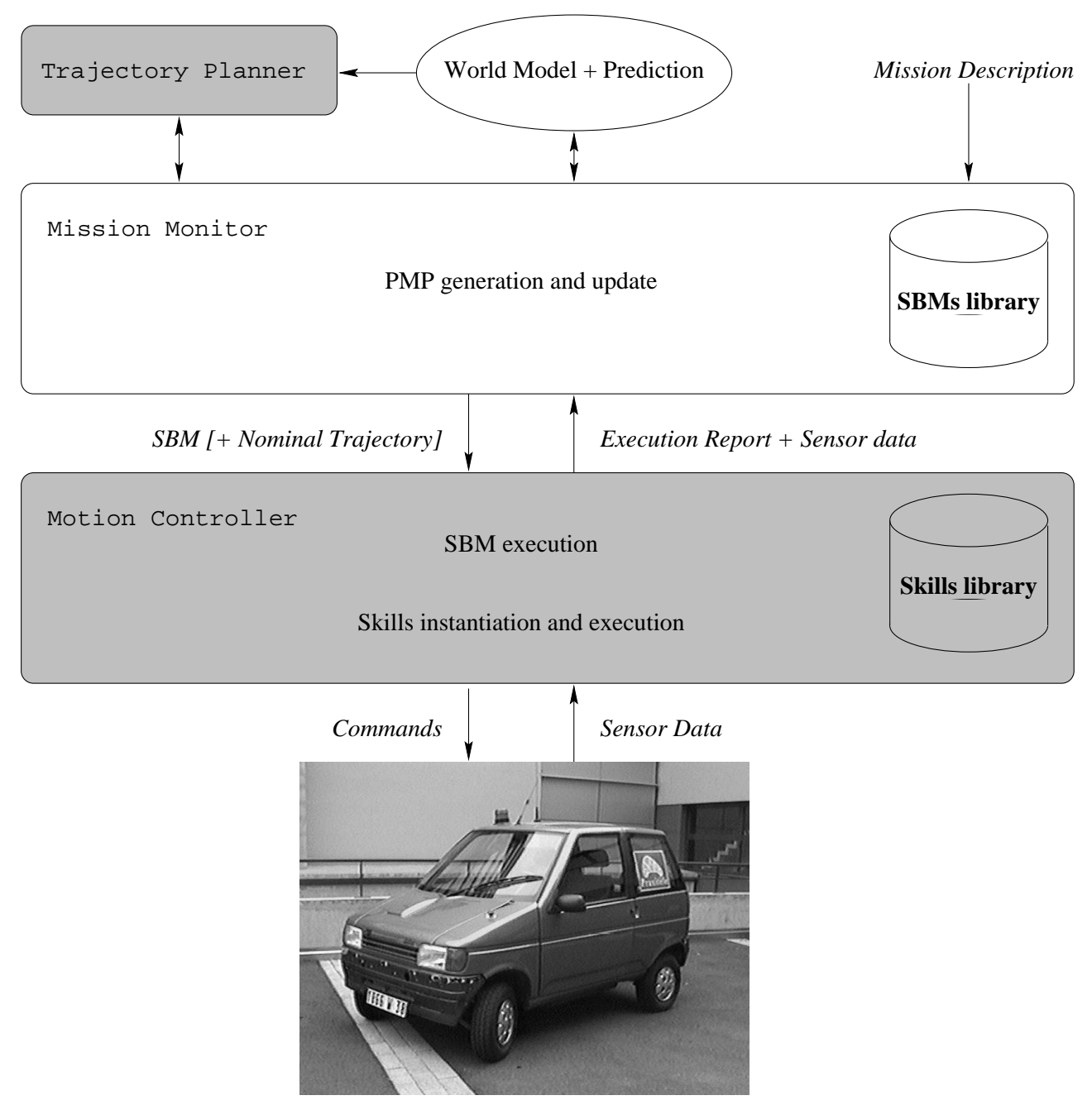

Figure 1: The overall control architecture.

knowledge, sensor data, output of other modules, etc.). Script parametres fill in the details of the script steps and permit to deal easily with the current task conditions.

The introduction of SBM was motivated by the observation that the kind of motion task that a vehicle has to perform can usually be described as a series of simple steps (a script). A SBM is a script, it combines control and sensing skills. Skills are elementary functions with real-time abilities: sensing skills are functions processing sensor data whereas control 
skills are control programs (open or closed loop) that generate the appropriate commands for the vehicle. Control skills may use data provided directly by the sensors or by the sensing skills.

The idea of combining basic real-time skills to build a plan in order to perform a given task can be found in other control architectures ( $c f . \S 7)$; they permit to obtain robust, flexible and reactive behaviours. SBMs can be seen as "meta-skills", their novelty is that they permit to encapsulate high-level expert human knowledge and heuristics about how to perform a specific motion task ( $c f . \S 5)$. Accordingly they permit to reduce the planning effort required to address a given motion task, thus improving the overall response-time of the system, while retaining the good properties of a skill-based architecture, i.e. robustness, flexibility and reactivity.

The control architecture features two main components, the mission monitor and the motion controller, that are described afterwards.

\subsection{Mission Monitor}

When given a mission description, e.g. "go park at location l", the mission monitor (MN) generates a parameterized motion plan (PMP) which is a set of generic sensor-based manœuvres (SBM) possibly completed with nominal trajectories. The SBMs are selected from a SBM library. A SBM may require a nominal trajectory (it is the case of the "Follow Trajectory" SBM). A nominal trajectory is a continuous time-ordered sequence of (position, velocity) of the vehicle that represents a theoretically safe and executable trajectory, i.e. a collision-free trajectory which satisfies the kinematic and dynamic constraints of the vehicle. Such trajectories are computed by the trajectory planner by using:

- an a priori known or acquired model of the vehicle environment,

- the current sensor data (e.g. position and velocity of the moving obstacles, and

- a world prediction that gives the most likely behaviours of the moving obstacles.

Trajectory planning is detailed in $\S 4$. The current SBM with its nominal trajectory is passed to the motion controller for its reactive execution.

\section{$2.2 \quad$ Motion Controller}

The goal of the Motion Controller (MC) is to execute in a reactive way the current SBM of the PMP. For that purpose, the current SBM is instantiated according to the current execution context, i.e. the variable parametres of the SBM are set by using the a priori known or sensed information available at the time, e.g. road curvature, available lateral and longitudinal space, velocity and acceleration bounds, distance to an obstacle, etc. As mentioned above, a SBM combines control and sensing skills that are either control programs or sensor data processing functions. It is up to $\mathrm{MC}$ to control and coordinate the execution of the different skills required. The sequence of control skills that is executed for a given 
SBM is determined by the events detected by the sensor skills. When an event that cannot be handled by the current SBM happens, MC reports a failure to MN which updates PMP either by applying a replanning procedure (time permitting), or by selecting in real-time a SBM adapted to the new situation.

\section{Model of the Vehicle}

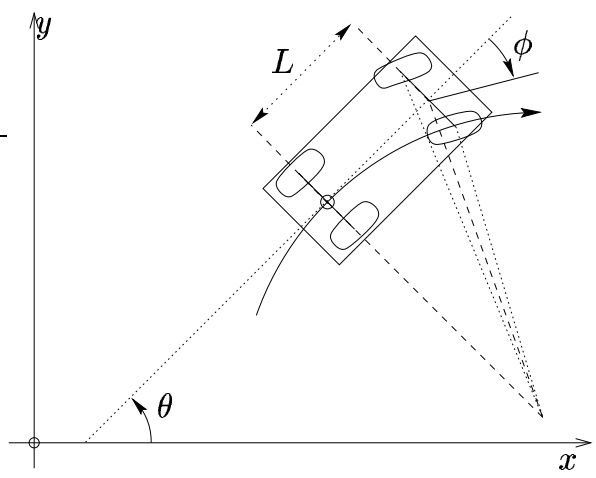

Figure 2: Model of a car-like vehicle.

A car-like vehicle is modelled as a rigid body moving on the plane. It is supported by four wheels making point contact with the ground, it has two rear wheels and two directional front wheels. The model of a car-like vehicle that is used is depicted in Fig. 2. The configuration, i.e. the position and orientation of the vehicle, are characterized by the triple $q=(x, y, \theta)$ where $x=x(t)$ and $y=y(t)$ are the coordinates of the rear axle midpoint and $\theta=\theta(t)$ the orientation of the vehicle, i.e. the angle between the $x$ axis and the main axis of the vehicle. The motion of the vehicle is described by the following equations:

$$
\left\{\begin{array}{l}
\dot{x}=v \cos \phi \cos \theta \\
\dot{y}=v \cos \phi \sin \theta \\
\dot{\theta}=\frac{v}{L} \sin \phi
\end{array}\right.
$$

where $\phi=\phi(t)$ is the steering angle, i.e. the average orientation of the two front wheels of the vehicle. $v=v(t)$ is the locomotion velocity of the front axle midpoint and $L$ is the wheelbase. $(\phi, v)$, the steering angle and locomotion velocity, are the two control commands of the vehicle. Since the steering angle of a car is mechanically limited, the following constraint also holds (maximum curvature constraint):

$$
|\phi| \leq \phi_{\max }
$$


Eqs. (1) correspond to a system with non-holonomic kinematic constraints because they involve the derivatives of the coordinates of the vehicle and are non-integrable [17]. They are valid for a vehicle moving on flat ground with perfect rolling assumption (no slippage between the wheels and the ground) at relatively low speed. For high-speed motions, the dynamics of the vehicle must also be considered. In the current implementation of the architecture, only velocity and acceleration bounds are taken into account.

\section{$4 \quad$ Trajectory Planning}

As mentioned earlier, trajectory planning is an important function in the control architecture proposed. Its purpose is to compute a nominal trajectory leading the vehicle to its goal. A trajectory is a continuous time-ordered sequence of states, i.e. (configurations, velocity) pairs, between the current state of the vehicle and its goal. A trajectory must be collision-free and satisfy the kinematic and dynamic constraints of the vehicle.

In order to plan a trajectory that avoids the moving obstacles of the environment, the knowledge of their future behaviour is required. In most cases, this information is not a priori known. An estimation of the most likely behaviour of the moving obstacles is provided by the prediction function $(c f . \S 2)$.

Trajectory planning for car-like vehicles in dynamic environments remains an open problem and a practical solution to this intricate problem is presented in this section.

\subsection{Outline of the Approach}

The motion of a vehicle is subject to several types of constraints and the nominal trajectory has to respect them. These constraints are:

- Kinematic constraints: a wheeled car-like vehicle is subject to kinematic constraints, called non-holonomic, that restricts the geometric shape of its motion. Such a vehicle can move only in a direction which is perpendicular to its rear wheel axle (non-steering wheels) and its turning radius is lower-bounded.

- Dynamic constraints: these constraints arise because of the dynamics of the vehicle and the capabilities of its actuators (engine power, braking force, ground-wheel interaction, etc.). They restrict the accelerations and velocities of the vehicle.

- No collision constraints: collision with stationary and moving obstacles of the environment are forbidden.

A trajectory is a time-ordered sequence of states $(q, \dot{q})$. It can be represented also by a geometric path and a velocity profile along this path. Because of the intrinsic complexity of trajectory planning ( $C f$. [17] for complexity issues), the trajectory planner addresses the problem at hand in two complementary steps of lesser complexity:

$\mathrm{RR} \mathrm{n}^{\circ} 3552$ 
1. Path planning: a geometric path leading the vehicle to its goal is computed. It is collision-free with the stationary obstacles of the environment and it respects the nonholonomic kinematic constraints of the vehicle.

2. Velocity planning: the velocity profile of the vehicle along its path is computed; this profile respects the dynamic constraints of the vehicle and yields no collisions between the vehicle and the moving obstacles of the environment.

Note that velocity planning requires the knowledge of the future behaviour of the moving obstacles; this information is provided by the prediction function.
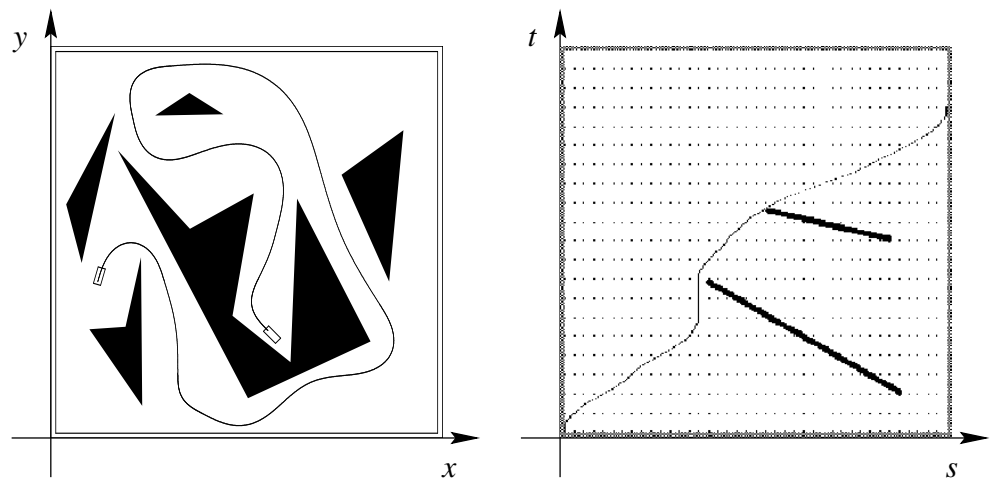

Figure 3: (a) Path planning and (b) velocity planning.

Path planning is illustrated in the left-hand side of Fig. 3. It depicts an example path between two configurations. This collision-free path is a continuous curve whose curvature is upper-bounded so as to respect the kinematic constraints of a car-like vehicle. The right-hand side of Fig. 3 illustrates velocity planning: it depicts a space-time diagram (the horizontal axis being the position along the path and the vertical one the time dimension). The curve represents the motion of the vehicle through time whereas the thick black lines are the traces left by moving obstacles when they cross the path of the vehicle.

The next two sections respectively present the path planning and the velocity planning steps.

\subsection{Path Planning}

As mentioned earlier, a car-like vehicle is subject to non-holonomic kinematic constraints: it can move only along a direction perpendicular to its rear wheels axle (continuous tangent direction), and its turning radius is lower-bounded (maximum curvature). In the past ten years, numerous works, e.g. $[3,18,31]$, have tackled the problem of computing feasible paths for this type of vehicle. Almost all of them compute paths made up of circular arcs connected 

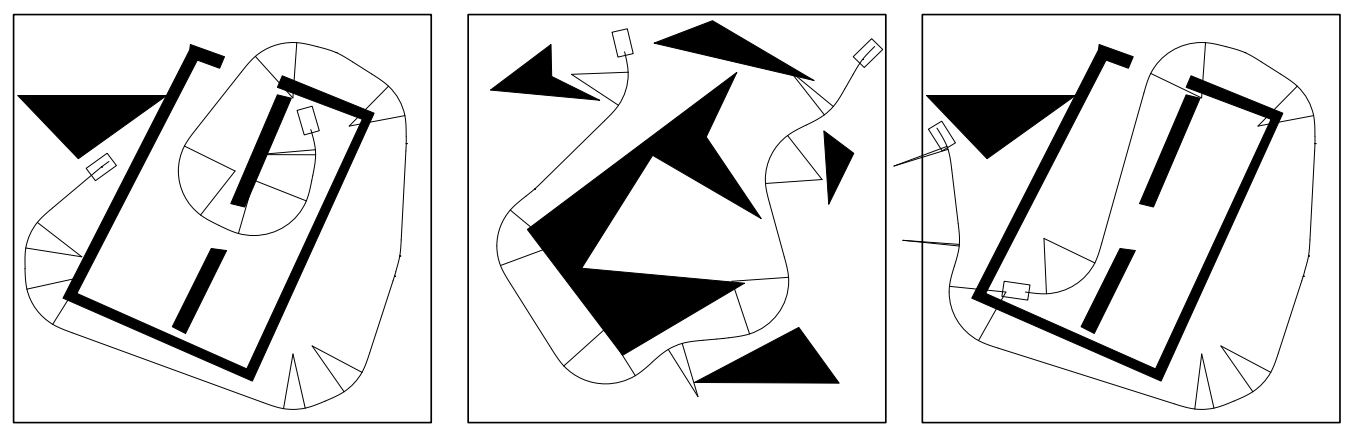

Figure 4: Examples of continuous curvature paths.

with tangential line segments. The key reason for that is that these paths are the shortest one that respect the non-holonomic kinematic constraints of such a vehicle [7, 26]. However their curvature profile is not continuous. Accordingly a vehicle following such a path has to stop at each curvature discontinuity, i.e. at each transition between a segment and an arc, in order to reorient its front wheels. This is hardly acceptable for a vehicle driving on the road. A solution to this problem is therefore to plan paths with a continuous curvature profile. In addition, a constraint on the curvature derivative is introduced; it is upper-bounded so as to reflect the fact that the vehicle can only reorient its front wheels with a finite velocity.

Addressing a similar problem (but without the maximum curvature constraint), [4] proves that the shortest path between two vehicle's configurations is made up of line segments and clothoids ${ }^{1}$ of maximum curvature derivative. Unfortunately, [16] later proved that these shortest paths are, in the general case, made up of an infinity of clothoids. These results also apply to the problem including the maximum curvature constraint. Therefore, in order to come up with a practical solution to the problem at hand, a set of paths that contain at most eight parts, each part being either a line segment, a circular arc, or a clothoid, has been defined. These paths have a continuous curvature profile, and an upper-bounded curvature and curvature derivative. They are used to design a local path planner, i.e. a non-complete collision-free path planner, which in turn is embedded in a global path planning scheme. The result is the first path planner for a car-like vehicle that generates collision-free paths with continuous curvature and upper-bounded curvature and curvature derivative. The reader is referred to [28] for a complete presentation of the continuous curvature path planner. Various experimental results are depicted in Fig. 4.

\subsection{Velocity Planning}

Given the nominal path generated by the path planner, the problem is to determine the trajectory of the vehicle along this path, i.e. its velocity profile; this profile must respect

\footnotetext{
${ }^{1} \mathrm{~A}$ clothoid is a curve whose curvature is a linear function of its arc length.
} 


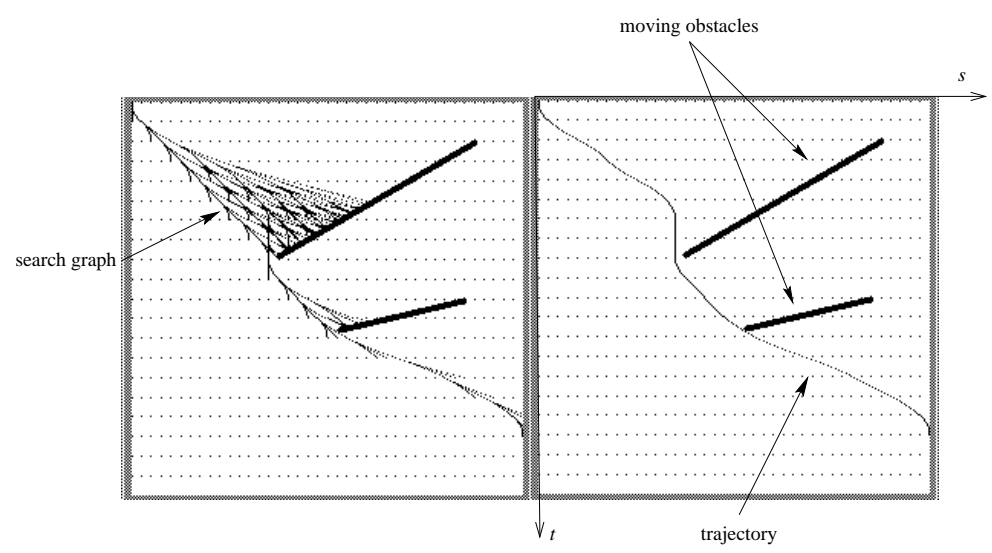

Figure 5: An example of velocity planning.

the dynamic constraints of the vehicle and yields no collision between the vehicle and the moving obstacles of the environment.

To address these two issues, i.e. moving obstacles and dynamic constraints, the concept of state-time space, has been introduced. It stems from two concepts that have been used before in order to deal respectively with moving obstacles and dynamic constraints, namely the concepts of configuration-time space [8], and state space, i.e. the space of the configuration parameters and their derivatives. Merging these two concepts leads naturally to state-time space, i.e. the state space augmented of the time dimension. In this framework, the constraints imposed by both the moving obstacles and the dynamic constraints are represented by static forbidden regions of state-time space. Besides a trajectory maps to a curve in state-time space hence trajectory planning in dynamic workspaces simply consists in finding a curve in state-time space, i.e. a continuous sequence of state-times between the current state of the vehicle and a goal state. Such a curve must obviously respect additional constraints due to the fact that time is irreversible and that velocity and acceleration constraints translate to geometric constraints on the slope and the curvature along the time dimension. However it is possible to extend previous methods for path planning in configuration space in order to solve the problem at hand. In particular, a method derived from the one originally presented in [6] has been designed to solve the problem at hand. It follows the paradigm of near-time-optimization: the search for the solution trajectory is performed over a restricted set of canonical trajectories hence the near-time-optimality of the solution. These canonical trajectories are defined as having piecewise constant acceleration that change its value at given times. Besides the acceleration is selected so as to be either minimum, null or maximum (bang controls). Under these assumptions, it is possible to transform the problem of finding the time-optimal canonical trajectory to finding the shortest path in a directed search graph embedded in the state-time space. 
An example of velocity planning is depicted in Fig. 5. There are two windows: a trace window showing the part of the search graph which has been explored and a result window displaying the final trajectory. Any such window represents the $s \times t$ plane (the position axis is horizontal while the time axis is vertical; the frame origin is at the upper-left corner). The thick black segments represent the trails left by the moving obstacles and the little dots are nodes of the underlying state-time search graph. The obstacles are assumed to keep a constant velocity. The vehicle starts from position 0 (upper-left corner) with a null velocity, it is to reach position 1 (right border) with a null velocity. The reader is referred to [9] and [10] for more details about velocity planning.

\section{Sensor-Based Manœuvres}

Recall that the control architecture proposed relies upon the concept of sensor-based manœuvres (SBM). At a given time instant, the vehicle is carrying out a particular SBM that has been instantiated to fit the current execution context (see $\S 2$ ). SBMs are general templates encoding the knowledge of how a given motion task is to be performed. They combine real-time functions, control and sensing skills, that are either control programs or sensor data processing functions.

This section describes the two SBMs that have been developed and integrated in the control architecture proposed: trajectory following and parallel parking. These two manœuvres have been implemented and successfully tested on a real automatic vehicle, the results of these experiments are presented in $\S 6$. The Orccad tool [30] has been selected to implement both SBMs and skills. The "robot procedure" formalism of Orccad represents SBMs while "robot-task" model skills. Robot procedures and robot tasks can both be represented as finite automata or transition diagrams. The "trajectory following" and "parallel parking" SBMs are depicted in Fig. 6 as transition diagrams. The control skills are represented by square boxes, e.g. "find parking place", whereas the sensing skills appear as predicates attached to the arcs of the diagram, e.g. "parking place detected", or conditional statements, e.g. "obstacle overtaken?". The next two sections describe how the two manœuvres illustrated in Fig. 6 operates.

\subsection{Trajectory Following}

The purpose of the trajectory following SBM is to allow the vehicle to follow a given nominal trajectory as closely as possible, while reacting appropriately to any unforeseen obstacle obstructing the way of the vehicle. Whenever such an obstacle is detected, the nominal trajectory is locally modified in real time, in order to avoid the collision. This local modification of the trajectory is done, in order to satisfy a set of different constraints: collision avoidance, time constraints, kinematic and dynamic constraints of the vehicle. In a previous approach, a fuzzy controller combining different basic behaviours (trajectory tracking, obstacle avoidance, etc.) were used to performed trajectory following [11]. However this approach proved 


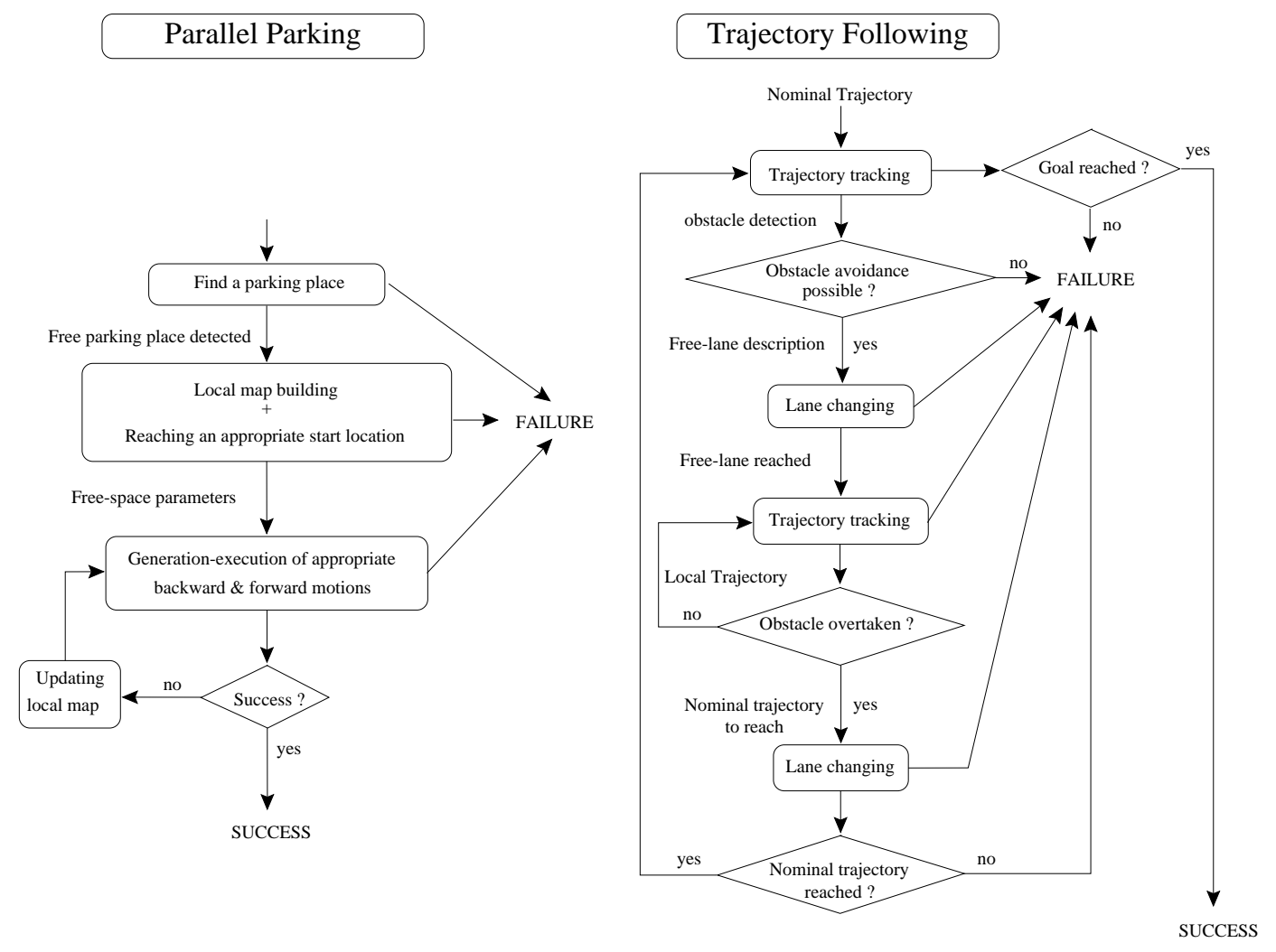

Figure 6: The "parallel parking" and "trajectory following" SBMs.

unsatisfactory: it would yields oscillating behaviours, and would not guarantee that all the abovementioned constraints were always satisfied.

The trajectory following SBM makes use of local trajectories to avoid the detected obstacles. These local trajectories allow the vehicle to move away from the obstructed nominal trajectory, and to catch up this nominal trajectory when the (stationary or moving) obstacle has been overtaken. All these local trajectories verify the motion constraints. This SBM relies upon two control skills, trajectory tracking and lane changing (cf. Fig. 6), that are detailed now.

\subsubsection{Trajectory Tracking}

The purpose of this control skill is to issue the control commands that will allow the vehicle to track a given nominal trajectory. Several control methods for non-holonomic robots have been proposed in the literature. The method described in [14] that ensures stable tracking 
of a feasible trajectory by a car-like robot has been selected for its simplicity and efficiency. The vehicle's control commands are of the following form :

$$
\begin{gathered}
\dot{\theta}=\dot{\theta}_{r e f}+v_{R, r e f}\left(k_{y} y_{e}+k_{\theta} \sin \theta_{e}\right), \\
v_{R}=v_{R, r e f} \cos \theta_{e}+k_{x} x_{e}
\end{gathered}
$$

where $q_{e}=\left(x_{e}, y_{e}, \theta_{e}\right)^{T}$ represents the error between the reference configuration $q_{r e f}$ and the current configuration $q$ of the vehicle $\left(q_{e}=q_{\text {ref }}-q\right), \dot{\theta}_{\text {ref }}$ and $v_{R, r e f}$ are the reference velocities, $v_{R}=v \cos \phi$ is the rear axle midpoint velocity, $k_{x}, k_{y}, k_{\theta}$ are positive constants (the reader is referred to [14] for full details about this control scheme).

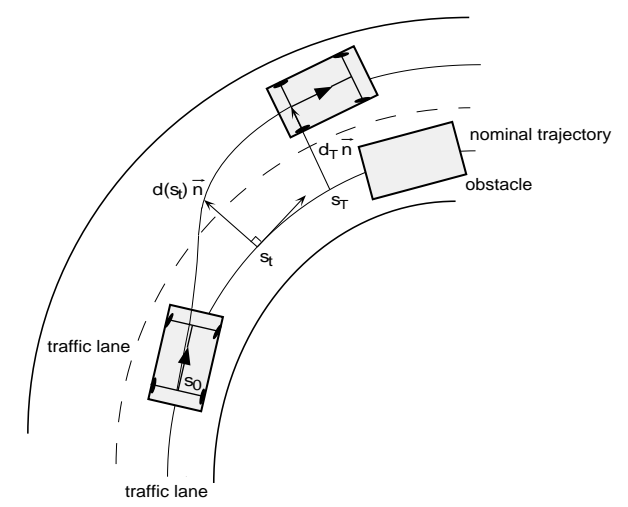

Figure 7: Generation of smooth local trajectories for avoiding an obstacle.

\subsubsection{Lane Changing}

This control skill is applied to execute a lane changing manœuvre. The lane changing is carried out by generating and tracking an appropriate local trajectory. Let $\mathcal{T}$ be the nominal trajectory to track, $d_{T}$ be the distance between $\mathcal{T}$ and the middle line of the free lane to reach, $s_{T}$ be the curvilinear distance along $\mathcal{T}$ between the vehicle and the obstacle (or the selected end point for the lane change), and $s=s_{t}$ be the curvilinear abscissa along $\mathcal{T}$ since the starting point of the lane change (cf. Fig. 7).

A feasible smooth trajectory for executing a lane change can be obtained using the following quintic polynomial (cf. [20]):

$$
d(s)=d_{T}\left(10\left(\frac{s}{s_{T}}\right)^{3}-15\left(\frac{s}{s_{T}}\right)^{4}+6\left(\frac{s}{s_{T}}\right)^{5}\right)
$$

$\mathrm{RR} \mathrm{n}^{\circ} 3552$ 
In this approach, the distance $d_{T}$ is supposed to be known beforehand. Then the minimal value required for $s_{T}$ can be estimated as follows:

$$
s_{T, \min }=\frac{\pi \sqrt{k d_{T}}}{2 \mathcal{C}_{\max }}
$$

where $\mathcal{C}_{\text {max }}$ stands for the maximum allowed curvature:

$$
\mathcal{C}_{\text {max }}=\min \left\{\frac{\tan \left(\phi_{\max }\right)}{L}, \frac{\gamma_{\text {max }}}{v_{R, \text { ref }}^{2}}\right\},
$$

$\gamma_{\max }$ is the maximum allowed lateral acceleration, and $k>1$ is an empirical constant (e.g.k $=1.17$ in our experiments).

At each time $t$ from the starting time $T_{0}$, the reference position $p_{\text {ref }}$ is translated along the vector $d\left(s_{t}\right) \cdot \vec{n}$, where $\vec{n}$ represents the unit normal vector to the nominal velocity vector along $\mathcal{T}$; the reference orientation $\theta_{\text {ref }}$ is converted into $\theta_{\text {ref }}+\arctan \left(\frac{\partial d}{\partial s}\left(s_{t}\right)\right)$, and the reference velocity $v_{R, r e f}$ is obtained using the following equation:

$$
v_{R, r e f}(t)=\frac{\operatorname{dist}\left(p_{r e f}(t), p_{\text {ref }}(t+\Delta t)\right)}{\Delta t},
$$

where dist stands for the Euclidean distance. As shown in Fig. 6, this type of control skill can also be used to avoid a stationary obstacle, or to overtake another vehicle. As soon as the obstacle has been detected by the vehicle, a value $s_{T, m i n}$ is computed according to (6) and compared with the distance between the vehicle and the obstacle. The result of this computation is used to decide which behaviour to apply: avoid the obstacle, slow down or stop. In this approach, an obstacle avoidance or overtaking manœuvre consists of lane changing manœuvre towards a collision-free "virtual" parallel trajectory(see Fig. 7). The lane changing skill operates the following way:

1. Generate a smooth local trajectory $\tau_{1}$ which connects $\mathcal{T}$ with a collision-free local trajectory $\tau_{2}$ "parallel" to $\mathcal{T}\left(\tau_{2}\right.$ is obtained by translating appropriately the involved piece of $\mathcal{T}$ ).

2. Track $\tau_{1}$ and $\tau_{2}$ until the obstacle has been overtaken.

3. Generate a smooth local trajectory $\tau_{3}$ which connects $\tau_{2}$ with $\mathcal{T}$, and track $\tau_{3}$.

\subsection{Parallel Parking}

Parallel parking comprises three main steps (cf. Fig. 6): localizing a free parking place, reaching an appropriate start location with respect to the parking place, and performing the parallel parking manœuvre using iterative backward and forward motions until the vehicle is parked. During the first step, the vehicle moves slowly along the traffic lane and uses its 




Figure 8: Situation at the beginning of a parallel parking manœuvre.

range sensors to build a local map of the environment and detect obstacles. The local map is used to determine whether free parking space is available to park the vehicle.

A typical situation at the beginning of a parallel parking manœuvre is depicted in Fig. 8. The autonomous vehicle $A 1$ is in the traffic lane. The parking lane with parked vehicles $B 1$, $B 2$ and a parking place between them is on the right-hand side of $A 1$. $L 1$ and $L 2$ are respectively the length and width of $A 1$, and $D 1$ and $D 2$ are the distances available for longitudinal and lateral displacements of $A 1$ within the place. $D 3$ and $D 4$ are the longitudinal and lateral displacements of the corner $A 13$ of $A 1$ relative to the corner $B 24$ of $B 2$.

Distances $D 1, D 2, D 3$ and $D 4$ are computed from data obtained by the sensor systems. The length $(D 1-D 3)$ and wide $(D 2-D 4)$ of the free parking place are compared with the length $L 1$ and width $L 2$ of $A 1$ in order to determine whether the parking place is sufficiently large.

During parallel parking, iterative low-speed backward and forward motions with coordinated control of the steering angle and locomotion velocity are performed to produce a lateral displacement of the vehicle into the parking place. The number of such motions depends on the distances $D 1, D 2, D 3, D 4$ and the necessary parking depth which depends on the width $L 2$ of the vehicle $A 1$. The start and end orientations of the vehicle are the same for each iterative motion.

For the $i$-th iterative motion (but omitting the index " $i$ "), let the start coordinates of the vehicle be $x_{0}=x(0), y_{0}=y(0), \theta_{0}=\theta(0)$ and the end coordinates be $x_{T}=x(T), y_{T}=y(T)$, $\theta_{T}=\theta(T)$, where $T$ is duration of the motion. The "parallel parking" condition means that

$$
\theta_{0}-\delta_{\theta}<\theta_{T}<\theta_{0}+\delta_{\theta},
$$

where $\delta_{\theta}>0$ is a small admissible error in orientation of the vehicle.

The following control commands of the steering angle $\phi$ and locomotion velocity $v$ provide the parallel parking manœuvre [24]:

$$
\begin{array}{ll}
\phi(t)=\phi_{\max } k_{\phi} A(t), & 0 \leq t \leq T, \\
v(t)=v_{\max } k_{v} B(t), & 0 \leq t \leq T,
\end{array}
$$


where $\phi_{\max }>0$ and $v_{\max }>0$ are the admissible magnitudes of the steering angle and locomotion velocity respectively, $k_{\phi}= \pm 1$ corresponds to a right side $(+1)$ or left side $(-1)$ parking place relative to the traffic lane, $k_{v}= \pm 1$ corresponds to forward $(+1)$ or backward $(-1)$ motion,

$$
\begin{aligned}
& A(t)= \begin{cases}1, & 0 \leq t<t^{\prime}, \\
\cos \frac{\pi\left(t-t^{\prime}\right)}{T^{*}}, & t^{\prime} \leq t \leq T-t^{\prime}, \\
-1, & T-t^{\prime}<t \leq T,\end{cases} \\
& B(t)=0.5\left(1-\cos \frac{4 \pi t}{T}\right), \quad 0 \leq t \leq T,
\end{aligned}
$$

where $t^{\prime}=\frac{T-T^{*}}{2}, T^{*}<T$. The shape of the type of paths that corresponds to the controls (12) and (13) is shown in Fig. 9.

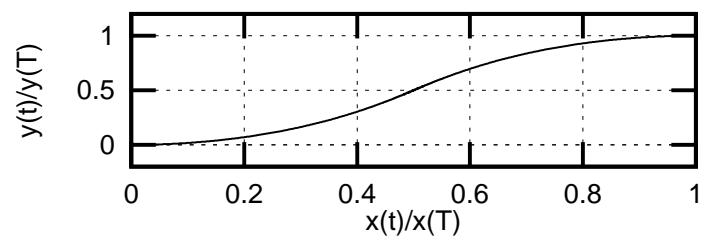

Figure 9: Shape of a parallel parallel forward/backward motion.

The commands (10) and (11) are open-loop in the $(x, y, \theta)$-coordinates. The steering wheel servo-system and locomotion servo-system must execute the commands (10) and (11), in order to provide the desired $(x, y)$-path and orientation $\theta$ of the vehicle. The resulting accuracy of the motion in the $(x, y, \theta)$-coordinates depends on the accuracy of these servosystems. Possible errors are compensated by subsequent iterative motions.

For each pair of successive motions $(i, i+1)$, the coefficient $k_{v}$ in (11) has to satisfy the equation $k_{v, i+1}=-k_{v, i}$ that alternates between forward and backward directions. Between successive motions, when the velocity is null, the steering wheels turn to the opposite side in order to obtain a suitable steering angle $\phi_{\max }$ or $-\phi_{\max }$ to start the next iterative motion.

In this way, the form of the commands (10) and (11) is defined by (12) and (13) respectively. In order to evaluate (10)-(13) for the parallel parking manœuvre, the durations $T^{*}$ and $T$, the magnitudes $\phi_{\max }$ and $v_{\max }$ must be known.

The value of $T^{*}$ is lower-bounded by the kinematic and dynamic constraints of the steering wheel servo-system. When the control command (10) is applied, the lower bound of $T^{*}$ is

$$
T_{\min }^{*}=\pi \max \left\{\frac{\phi_{\max }}{\dot{\phi}_{\max }}, \sqrt{\frac{\phi_{\max }}{\ddot{\phi}_{\max }}}\right\},
$$

where $\dot{\phi}_{\max }$ and $\ddot{\phi}_{\max }$ are the maximal admissible steering rate and acceleration respectively for the steering wheel servo-system. The value of $T_{m i n}^{*}$ gives duration of the full turn of the steering wheels from $-\phi_{\max }$ to $\phi_{\max }$ or vice versa, i.e. one can choose $T^{*}=T_{\min }^{*}$. 
The value of $T$ is lower-bounded by the constraints on the velocity $v_{\max }$ and acceleration $\dot{v}_{\text {max }}$ and by the condition $T^{*}<T$. When the control command (11) is applied, the lower bound of $T$ is

$$
T_{\min }=\max \left\{\frac{2 \pi v^{\prime}(D 1)}{\dot{v}_{\max }}, T^{*}\right\},
$$

where $v^{\prime}(\mathrm{D} 1) \leq v_{\max }$, empirically-obtained function, serves to provide a smooth motion of the vehicle when the available distance $D 1$ is small.

The computation of $T$ and $\phi_{\max }$ aims to obtain the maximal values such that the following "longitudinal" and "lateral" conditions are still satisfied:

$$
\begin{aligned}
& \left|\left(x_{T}-x_{0}\right) \cos \theta_{0}+\left(y_{T}-y_{0}\right) \sin \theta_{0}\right|<D 1, \\
& \left|\left(x_{0}-x_{T}\right) \sin \theta_{0}+\left(y_{T}-y_{0}\right) \cos \theta_{0}\right|<D 2 .
\end{aligned}
$$

Using the maximal values of $T$ and $\phi_{\max }$ assures that the longitudinal and, especially, lateral displacement of the vehicle is maximal within the available free parking space. The computation is carried out on the basis of the model (1) when the commands (10) and (11) are applied. In this computation, the value of $v_{\max }$ must correspond to a safety requirement for parking manœuvres (e.g. $v_{\max }=0.75 \mathrm{~m} / \mathrm{s}$ was found empirically).

At each iteration $i$ the parallel parking algorithm is summarized as follows:

1. Obtain available longitudinal and lateral displacements $D 1$ and $D 2$ respectively by processing the sensor data.

2. Search for maximal values $T$ and $\phi_{\max }$ by evaluating the model (1) with controls (10), (11) so that conditions (16), (17) are still satisfied.

3. Steer the vehicle by controls (10), (11) while processing the range data for collision avoidance.

4. Obtain the vehicle's location relative to environmental objects at the parking place. If the "parked" location is reached, stop; else, go to step 1.

When the vehicle $A 1$ moves backwards into the parking place from the start location shown in Fig. 8, the corner $A 12$ (front right corner of the vehicle) must not collide with the corner $B 24$ (front left corner of the place). The start location must ensure that the subsequent motions will be collision-free with objects limiting the parking place. To obtain a convenient start location, the vehicle has to stop at a distance $D 3$ that will ensure a desired minimal safety distance $D 5$ between the vehicle and the nearest corner of the parking place during the subsequent backward motion. The relation between the distances $D 1, D 2, D 3, D 4$ and $D 5$ is described by a function $\mathcal{F}(D 1, D 2, D 3, D 4, D 5)=0$. This function can not be expressed in closed form, but it can be estimated for a given type of vehicle by using the model (1) when the commands (10) and (11) are applied. The computations are carried out off-line and the results are stored in a look-up table which is used on-line, to obtain an estimate of $D 3$ corresponding to a desired minimal safety distance $D 5$ for given $D 1, D 2$ and $D 4$ [23].

$\mathrm{RR} \mathrm{n}^{\circ} 3552$ 
When the necessary parking "depth" has been reached, clearance between the vehicle and the parked ones is provided, i.e. the vehicle moves forwards or backwards so as to be in the middle of the parking place between the two parked vehicles.

\section{Experimental Results}

The approach described in the report has been implemented and tested on our experimental automatic vehicle (a modified Ligier electric car). This vehicle is equipped with the following capabilities: (1) - a sensor unit to measure relative distances between the vehicle and environmental objects, (2) - a servo unit to control the steering angle and the locomotion velocity, and (3) - a control unit that processes data from the sensor and servo units in order to "drive" the vehicle by issuing appropriate servo commands. This vehicle can either be manually driven, or it can move autonomously using the control unit based on a Motorola VME162-CPU board and a transputer net. A VxWorks real-time operating system is used. The sensor unit of the vehicle makes use of a belt of ultrasonic range sensors (Polaroid 9000) and of a linear CCD-camera. The servo unit consists of a steering wheel servo-system, a locomotion servo-system for forward and backward motions, and a braking servo-system to slow down and stop the vehicle. The steering wheel servo-system is equipped with a direct current motor and an optical encoder to measure the steering angle. The locomotion servo-system of the vehicle is equipped with a $12 \mathrm{~kW}$ asynchronous motor and two optical encoders located onto the rear wheels (for odometry data). The vehicle has an hydraulic braking servo-system. The Motion Controller monitors the current steering angle, locomotion velocity, travelled distance, coordinates of the vehicle and range data from the environment, calculates an appropriate local trajectory and issues the required servo commands. The Motion Controller has been implemented using the Orccad software tools [30] running on a Sun workstation. The compiled code is transmitted via Ethernet to the VME162-CPU board.

The experimental car is equipped with 14 ultrasonic range sensors (Polaroid 9000), 8 of them - a minimal configuration - are used for the current version of the automatic parking system - 3 ultrasonic sensors are in the front of the car (looking in the forward direction), two sensors are situated on each side of the car and one ultrasonic sensor is in the rear of the car (looking in the backward direction). The measurement range is $0.5 \mathrm{~m}-10.0$ $\mathrm{m}$, the sampling rate is $60 \mathrm{~ms}$. The sensors are activated sequentially (four sensors are emitting/receiving signals at each instant - one for each side of the car). This sensor system is intended for testing the control algorithms only and for low-speed motion only. Certainly, a more complex sensor system, e.g.a combination of vision and ultrasonic sensors, must be use to ensure reliable operation in a dynamic environment.

An experimental run of the follow trajectory SBM with obstacle avoidance on circular road (roundabout) is shown in Fig. 10. In this experiment, the Ligier vehicle follows a nominal trajectory along the curved traffic lane, and it finds on its way another vehicle moving at a lower velocity (see Fig. 10a). When the moving obstacle is detected, a local trajectory for a right lane change is generated by the system, and the Ligier performs the 

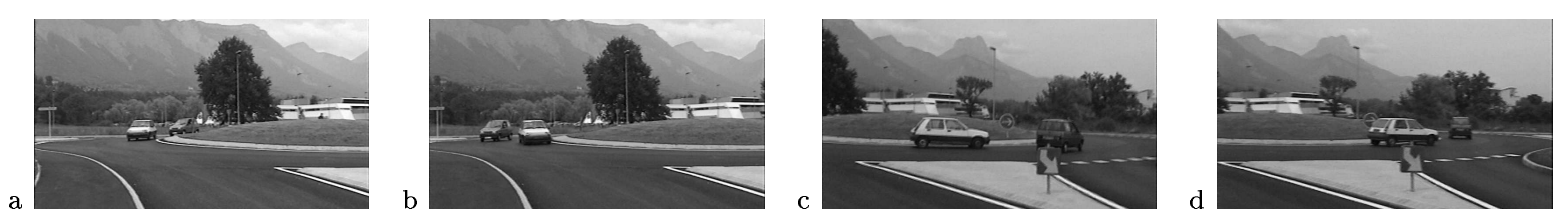

Figure 10: Snapshots of trajectory following with obstacle avoidance in a roundabout: (a) following the nominal trajectory, (b) lane changing to the right and overtaking, (c) lane changing to the left, (d) catching up with the nominal trajectory.
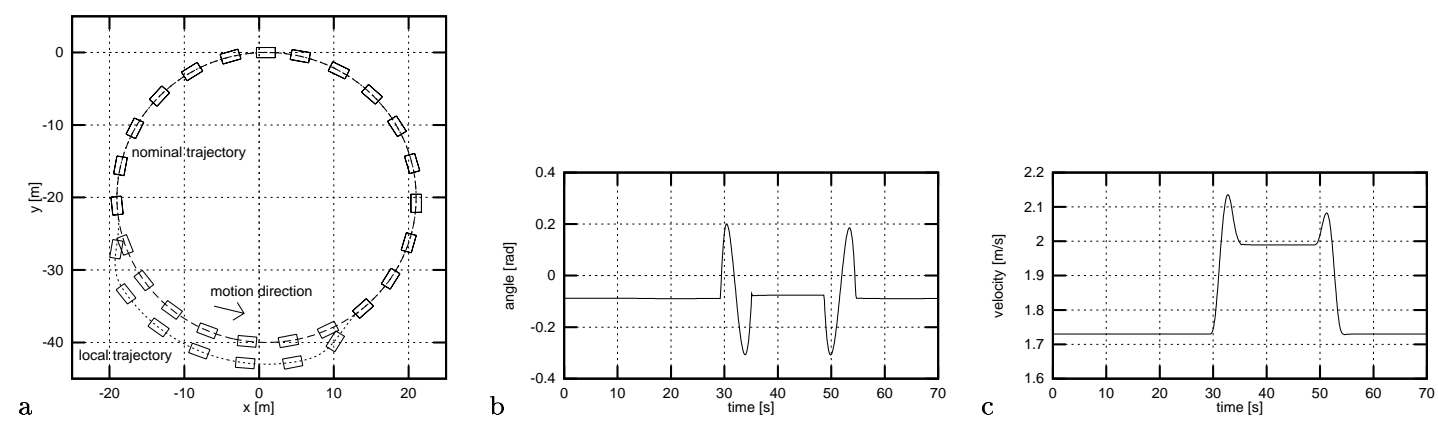

Figure 11: Motion and control commands in the "roundabout" scenario: (a) motion, (b) steering angle and (c) velocity controls applied.

lane changing manœuvre, as illustrated in Fig.10b. Afterwards, the Ligier moves along a trajectory parallel to its nominal trajectory, and a left lane change is performed as soon as the obstacle has been overtaken (Fig. 10c). Finally the Ligier catches up its nominal trajectory, as illustrated in Fig. 10d.

The corresponding motion of the vehicle is depicted in Fig. 11a. The steering and velocity controls applied during this manœuvre are shown in Fig. 11b and Fig. 11c. It can be noticed in this example that the velocity of the vehicle has increased when moving along the local "parallel" trajectory (Fig. 11c); this is due to the fact that the vehicle has to satisfy the time constraints associated to its nominal trajectory.

An experimental run of the parallel parking SBM in a street is shown in Fig. 12. This manœuvre can be carried out in environments including moving obstacles, e.g. pedestrians or some other vehicles ( $c f$. the video [25]). In this experiment, the Ligier was manually driven to a position near the parking place, the driver started the autonomous parking mode and left the vehicle. Then, the Ligier moved forward autonomously in order to localize the parking place, obtained a convenient start location, and performed a parallel parking manœuvre. When, during this motion a pedestrian crosses the street in a dangerous proximity to the vehicle, as shown in Fig. 12a, this moving obstacle is detected, the Ligier slows down and stops to avoid the collision. When the way is free, the Ligier continues its forward motion. 

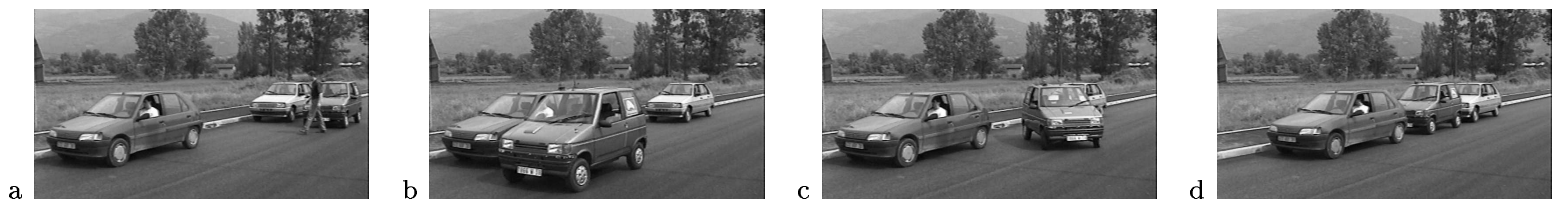

Figure 12: Snapshots of a parallel parking: (a) localizing a free parking place, (b) selecting an appropriate start location, (c) performing a backward parking motion; (d) completing the parallel parking.
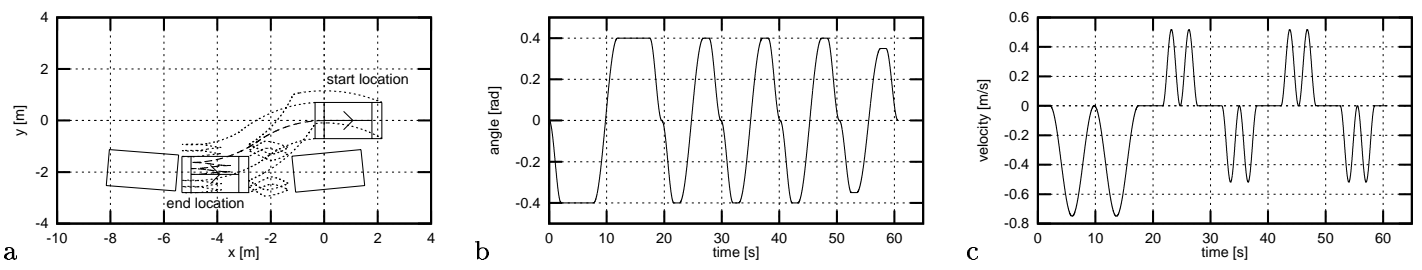

Figure 13: Motion and control commands in the parallel parking scenario: (a) motion, (b) steering angle and (c) velocity controls applied.

Range data is used to detect the parking bay. A decision to carry out the parking maneuver is made and a convenient start position for the initial backward movement is obtained, as shown in Fig. 12b. Then, the Ligier moves backwards into the bay, as shown in Fig. 12c. During this backward motion, the front human-driven vehicle starts to move backwards, reducing the length of the bay. The change in the environment is detected and taken into account. The range data shows that the necessary "depth" in the bay has not been reached, so further iterative motions are carried out until it has been reached. Then, the Ligier moves to the middle between the rear and front vehicles, as shown in Fig. 12d. The parallel parking maneuver is completed.

The corresponding motion of the vehicle is depicted in Fig. 13a where the motion of the corners of the vehicle and the midpoint of the rear wheel axle is plotted. The control commands (10) and (11) for parallel parking into a parking place situated at the right side of the vehicle are shown in Fig. 13b and Fig. 13c respectively. The length of the vehicle is $L 1=2.5 \mathrm{~m}$, the width is $L 2=1.4 \mathrm{~m}$, and the wheelbase is $L=1.785 \mathrm{~m}$. The available distances are $D 1=4.9 \mathrm{~m}, D 2=2.7 \mathrm{~m}$ relative to the start location of the vehicle. The lateral distance $D 4=0.6 \mathrm{~m}$ was measured by the sensor unit. The longitudinal distance $D 3=0.8 m$ was estimated so as to ensure the minimal safety distance $D 5=0.2 m$. In this case, five iterative motions are performed to park the vehicle. As seen in Fig. 13, the durations $T$ of the iterative motions, magnitudes of the steering angle $\phi_{\max }$ and locomotion velocity $v_{\max }$ correspond to the available displacements $D 1$ and $D 2$ within the parking place (e.g.the values of $T, \phi_{\max }$ and $v_{\max }$ differ for the first and last iterative motion). 


\section{Related Works}

As mentioned in $\S 1$, motion autonomy has been a long standing issue in Robotics hence the important number of works presenting control architectures for robot systems. All these architectures are not reviewed here, the main trends are indicated instead.

Three main functions are to be found in any control architecture: perception, decision and action (hence the 'perception-decision-action' paradigm). After a careful examination of the existing control architectures, it appears that, to some extent, the difference between them lies in the decision function. Two types of approaches of completely opposite philosophy have appeared:

- deliberative approaches: in this type of approach, complex models of the environment of the robot are built from sensory data or a priori knowledge. These models are then used to perform high-level reasoning, i.e. planning, in order to determine which action to undertake. Maintaining these models and reasoning about them is, in most cases, a time-consuming process that makes these methods unable to deal with dynamic and uncertain environments. [19, 21] and [32] are good examples of this type of control architectures.

- reactive approaches: the philosophy of this type of approach is just the opposite: they favor reactivity. The decision function is reduced to a minimum. Action follows perception closely, almost like a reflex. This type of approach is most appropriate to dynamic and uncertain environments since unexpected events can be dealt with as soon as they are detected by the sensors of the robot. One drawback however, high-level reasoning is very difficult to achieve (if not impossible). [5] is the canonical sensor-based control architecture; other examples are given in [15] or [33].

In an attempt to combine the advantages of both deliberative and reactive approaches, several authors have tried to combine high and low-level reasoning functions within a single control architecture. This idea permits to obtain hybrid control architectures with both high-level reasoning capabilities and reactivity.

The first hybrid architectures were obtained by simply putting together a deliberative and a reactive component. For instance, [2] integrates a simple motion planner to a reactive architecture whereas [13] sends the output of a task planner to a simple reactive execution controller: when a problem is detected at execution time, a reflex action is performed and the task planner is reinvoked. The performance of these approaches in terms of robustness, flexibility and reactivity are far from satisfactory. Better architectures have been proposed since, e.g. $[1,12]$ or $[29]$, they all combine three functional components:

- A set of elementary real-time functions (control loops, sensor data processing functions, etc.). A task is performed through the activation of such functions.

- A reactive execution mechanism that control and coordinates the execution of the real-time functions. 
- A decision module that produces the task plan and supervises its execution. It may react to events from the execution function.

The control architecture presented in this report clearly falls into this class of hybrid architectures. Skills are the real-time functions, the motion controller is the execution mechanism while the mission monitor is the decision module. With regard to these architectures, the main novelty of the approach proposed lies in the introduction of a meta-level of real-time functions, the sensor-based manœuvres, that encapsulate high-level expert human knowledge and heuristics about the motion tasks to be performed, that permit to reduce the planning effort required to address a given motion task and thus to improve the overall response-time of the system.

\section{Conclusion}

This report has presented an integrated control architecture endowing a car-like vehicle moving in a dynamic and partially known environment (the road network) with autonomous motion capabilities. Like most recent control architectures for autonomous robot systems, it combines three functional components: a set of basic real-time skills, a reactive execution mechanism and a decision module. The main novelty of the architecture proposed lies in the introduction of a fourth component akin to a meta-level of skills: the sensor-based manœuvres, i.e. general templates that encode high-level expert human knowledge and heuristics about how a specific motion task is to be performed. The concept of sensor-based manœuvres permit to reduce the planning effort required to address a given motion task, thus improving the overall response-time of the system, while retaining the good properties of a skill-based architecture, i.e. robustness, flexibility and reactivity.

After a general overview of the architecture proposed, the report has covered in more details the trajectory planning function (which is an important part of the decision module) and two types of sensor-based manœuvres: trajectory following and parallel parking. Experimental results with a real automatic car-like vehicle in different situations have been reported to demonstrate the efficiency of the approach. Future works will include the development and testing of other types of sensor-based manœuvres.

\section{Acknowledgements}

This work was partially supported by the Inria-Inrets ${ }^{2}$ Praxitèle programme on urban public transport [1994-1997], and the Inco-Copernicus ERB-IC15-CT96-0702 project "Multi-agent robot systems for industrial applications in the transport domain" [1997-1999]. The authors would like to thank E. Gauthier for his valuable contribution to the final version of the report.

${ }^{2}$ Institut National de Recherche sur les Transports et leur Sécurité. 


\section{References}

[1] R. Alami, R. Chatila, S. Fleury, M. Ghallab, and F. Ingrand. An architecture for autonomy. Int. Journal of Robotics Research, 17(4):315-337, April 1998.

[2] R. C. Arkin. Motor schema based navigation for a mobile robot. In Proc. of the IEEE Int. Conf. on Robotics and Automation, volume 1, pages 264-271, San Franciso, CA (US), 1987.

[3] J. Barraquand and J.-C. Latombe. On non-holonomic mobile robots and optimal maneuvering. Revue d'Intelligence Artificielle, 3(2):77-103, 1989.

[4] J.-D. Boissonnat, A. Cérézo, and J. Leblond. A note on shortest paths in the plane subject to a constraint on the derivative of the curvature. Research Report 2160, Inst. Nat. de Recherche en Informatique et en Automatique, Rocquencourt (FR), January 1994.

[5] R. A. Brooks. A robust layered control system for a mobile robot. In G. Shafer and J. Perl, editors, Readings in Uncertain Reasoning, pages 204-213. Morgan Kaufmann, 1990.

[6] J. Canny, B. Donald, J. Reif, and P. Xavier. On the complexity of kynodynamic planning. In Proc. of the IEEE Symp. on the Foundations of Computer Science, pages 306-316, White Plains, NY (USA), November 1988.

[7] L. E. Dubins. On curves of minimal length with a constraint on average curvature, and with prescribed initial and terminal positions and tangents. American Journal of Mathematics, 79:497-517, 1957.

[8] M. Erdmann and T. Lozano-Perez. On multiple moving objects. Algorithmica, 2:477$521,1987$.

[9] Th. Fraichard. Dynamic trajectory planning with dynamic constraints: a 'state-time space' approach. In Proc. of the IEEE-RSJ Int. Conf. on Intelligent Robots and Systems, volume 2, pages 1394-1400, Yokohama (JP), July 1993.

[10] Th. Fraichard and A. Scheuer. Car-like robots and moving obstacles. In Proc. of the IEEE Int. Conf. on Robotics and Automation, volume 1, pages 64-69, San Diego, CA (US), May 1994.

[11] Ph. Garnier and Th. Fraichard. A fuzzy motion controller for a car-like vehicle. In Proc. of the IEEE-RSJ Int. Conf. on Intelligent Robots and Systems, volume 3, pages 1171-1178, Osaka (JP), November 1996.

[12] E. Gat. On three-layer architectures. In D. Kortenkamp, R. P. Bonnasso, and R. Murphy, editors, Artificial Intelligence and Mobile Robots. MIT/AAAI Press, 1997.

$\mathrm{RR} \mathrm{n}^{\circ} 3552$ 
[13] E. Gat, M. G. Slack, D. P. Miller, and R. J. Firby. Path planning and execution monitoring for a planetary rover. In Proc. of the IEEE Int. Conf. on Robotics and Automation, volume 1, pages 20-25, Cincinatti, OH (US), 1990.

[14] Y. Kanayama, Y. Kimura, F. Miyazaki, and T. Noguchi. A Stable Tracking Control Method for a Non-Holonomic Mobile Robot. In Proc. of the IEEE-RSJ Int. Conf. on Intelligent Robots and Systems, Osaka (JP), November 1991.

[15] M. Khatib and R. Chatila. An Extended Potential Field Approach For Mobile Robot Sensor-Based Motions. In Proc. of Intelligent Autonomous Systems, pages 490-496, March 1995.

[16] V. Kostov and E. Degtiariova-Kostova. Some properties of clothoids. Research Report 2752, Inst. Nat. de Recherche en Informatique et en Automatique, December 1995.

[17] J. C. Latombe. Robot Motion Planning. Kluwer Academic Publishers, 1991.

[18] J.-P. Laumond, P. E. Jacobs, M. Taïx, and R. M. Murray. A motion planner for non-holonomic mobile robots. IEEE Trans. Robotics and Automation, 10(5):577-593, October 1994.

[19] H. P. Moravec. The stanford cart and the CMU rover. Proceedings of the IEEE, 71(7):872-884, 1983.

[20] W. L. Nelson. Continuous curvature paths for autonomous vehicles. In Proc. of the IEEE Int. Conf. on Robotics and Automation, volume 3, pages 1260-1264, Scottsdale, AZ (US), May 1989.

[21] N. J. Nilsson. Shakey the robot. Technical note 323, AI Center, SRI International, Menlo Park, CA (US), April 1984.

[22] M. Parent and P. Daviet. Automated urban vehicles: towards a dual mode PRT (Personal Rapid Transit). In Proc. of the IEEE Int. Conf. on Robotics and Automation, pages 3129-3134, Minneapolis, MN (US), April 1996.

[23] I. E. Paromtchik and C. Laugier. Autonomous parallel parking of a nonholonomic vehicle. In Proc. of the IEEE Int. Symp. on Intelligent Vehicles, pages 13-18, Tokyo (JP), September 1996.

[24] I. E. Paromtchik and C. Laugier. Motion generation and control for parking an autonomous vehicle. In Proc. of the IEEE Int. Conf. on Robotics and Automation, pages 3117-3122, Minneapolis, MN (US), April 1996.

[25] I. E. Paromtchik and Ch. Laugier. Automatic parallel car parking. In: VideoProceedings of the IEEE Int. Conf. on Robotics and Automation., April Albuquerque, NM (US)1997. Produced by Inst. Nat. de Recherche en Informatique et en AutomatiqueUnité de Communication et Information Scientifique ( $3 \mathrm{~min}$.). 
[26] J. A. Reeds and L. A. Shepp. Optimal paths for a car that goes both forwards and backwards. Pacific Journal of Mathematics, 145(2):367-393, 1990.

[27] E. Rich and K. Knight. Artificial Intelligence. McGraw-Hill, 1983.

[28] A. Scheuer and Th. Fraichard. Continuous-curvature path planning for car-like vehicles. In Proc. of the IEEE-RSJ Int. Conf. on Intelligent Robots and Systems, volume 2, pages 997-1003, Grenoble (FR), September 1997.

[29] R. G. Simmons. Structured control for autonomous robots. IEEE Trans. Robotics and Automation, 10(1):34-43, 1994.

[30] D. Simon, B. Espiau, E. Castillo, and K. Kapellos. Computer-Aided Design of a Generic Robot Controller Handling Reactivity and Real-Time Control Issues. In IEEE Transactions on Control Systems Technology, pages 213-229, December 1993.

[31] P. Švestka and M. H. Overmars. Coordinated motion planning for multiple car-like robots using probabilistic roadmaps. In Proc. of the IEEE Int. Conf. on Robotics and Automation, volume 2, pages 1631-1636, Nagoya (JP), May 1995.

[32] A. M. Waxman, J. Le Moigne, and B. Srinivasan. Visual Navigation of Roadways. In Proc. of the IEEE Int. Conf. on Robotics and Automation, pages 862-867, Saint Louis, MI (US), 1985.

[33] R. Zapata, B. Jouvencel, and P. Lepinay. Sensor-based Motion Control for Fast Mobile Robots. In IEEE Int. Workshop on Intelligent Motion Control, Istambul (TR), August 1990.

$\mathrm{RR} \mathrm{n}^{\circ} 3552$ 
Unit`e de recherche INRIA Lorraine, Technopôle de Nancy-Brabois, Campus scientifique, 615 rue du Jardin Botanique, BP 101, 54600 VILLERS LÈS NANCY

Unit'e de recherche INRIA Rennes, Irisa, Campus universitaire de Beaulieu, 35042 RENNES Cedex Unit'e de recherche INRIA Rhône-Alpes, 655, avenue de l'Europe, 38330 MONTBONNOT ST MARTIN Unit'e de recherche INRIA Rocquencourt, Domaine de Voluceau, Rocquencourt, BP 105, 78153 LE CHESNAY Cedex Unit'e de recherche INRIA Sophia-Antipolis, 2004 route des Lucioles, BP 93, 06902 SOPHIA-ANTIPOLIS Cedex

Éditeur

INRIA, Domaine de Voluceau, Rocquencourt, BP 105, 78153 LE CHESNAY Cedex (France) http://www.inria.fr ISSN 0249-6399 